\title{
A new route to the necessity of origin
}

\author{
Guy Rohrbaugh and Louis deRosset
}

July 20, 2004

\section{Introduction}

Saul Kripke (1980, 112-4) has claimed that there are necessary connections between material things and their material origins. His presentation of such necessity of origin theses included two paradigmatic examples: that it is impossible for a particular table to have been constructed from any hunk of matter other than that from which it was actually constructed, or for a particular human being to have originated in gametes other than those in which he or she actually did. Necessity of origin theses (henceforth, simply 'origin theses') are now standard examples of so-called metaphysical necessities, though unlike many other examples, they posit necessary connections between distinct individuals. Despite their familiarity, the conceptual grounds of origin theses are less clear than is usually supposed. Kripke's influential discussion provides only the briefest sketch of an argument. More explicit treatments have since been offered, but all of these turn out to be variations of just two strategies. J. L. Mackie and Penelope Mackie suggest that origin theses are grounded in a branching times model of necessity. Nathan Salmon, Colin McGinn, Graeme Forbes, and others suggest that such theses are grounded in a further principle, the sufficiency of origin. ${ }^{1}$ Both approaches depend on stronger assumptions than Kripke's original presentation appeared to call for, assumptions strong enough to engender a measure of skepticism in the philosophical community.

In this paper, we identify a third, overlooked approach to such theses, an argument from what we call independence principles. On this approach, origin theses are by-products of a more fundamental feature of the processes by which material objects are produced, their mutual independence. Independence principles are motivated, in turn, as a consequence of a plausible metaphysical

\footnotetext{
1 For the claim that origin theses are an upshot of the branching framework, see J. L. Mackie (1974) and P. Mackie (1998). Accounts that ground origin theses in the sufficiency of origin include McGinn (1976); Johnston (1977); Salmon (1979); Noonan (1983); and Forbes (1981, 1985, ch. 6). Forbes also makes use of a branching-worlds framework, but it plays no direct role in his argument for the origin thesis. Rather, it serves to support Forbes' sufficiency of origin thesis by restricting the range of worlds to which it applies. Both approaches develop suggestions found in footnotes 56 and 57 to Kripke's original discussion. The independence approach offered in this paper may constitute an interpretation of Kripke's argument in footnote 56 , but the textual evidence is unclear.
} 
principle governing such processes: their invulnerability to non-local prevention. We explain this approach and argue that it yields valid arguments for origin theses, is distinct from the two orthodox approaches, and proceeds from more plausible assumptions.

\section{The Locality of Prevention}

Origin theses express restrictions on possible processes of creation. They claim that certain logically possible productions, e.g., that of Elizabeth Windsor from the Trumans, are not in fact possible. But why shouldn't this be possible? Finding a principled answer to this question requires consideration of a broader one: What does it take to prevent the production of a particular material object from a particular hunk of raw material? Consider Kripke's example of a table, $T_{1}$, and the hunk of wood, $H_{1}$, from which it was actually produced. It is a contingent fact that $H_{1}$ gives rise to $T_{1}$. There are many ways it might not have come to pass. We might have made $H_{1}$ into a chair; we might have burned $H_{1}$ for warmth; we might have seized the means of table production; we might just have decided to leave $H_{1}$ alone. In all of these cases, some factor prevents the production of $T_{1}$ from $H_{1}$. The factors that prevent $T_{1}$ 's production in each case are those divergences from the actual circumstances responsible for $T_{1}$ 's not coming from $H_{1}$ in that case. ${ }^{2}$

We should notice an important feature of these examples. Each factor which prevents $T_{1}$ from eventuating makes a difference to $H_{1}$ or the people and tools involved in the productive effort. In this sense, the preventions are local. What makes no difference to the existence of $H_{1}$ or the process by which $T_{1}$ actually came from $H_{1}$ is irrelevant to $T_{1}$ 's production and cannot interfere. These reflections suggest that processes of table-creation are governed by a principle of locality of prevention: any case in which some factor prevents the production of $T_{1}$ from $H_{1}$ must differ from actual circumstances with respect to properties of either $H_{1}$ or other elements of the process by which $T_{1}$ actually emerged. ${ }^{3}$

The locality of prevention is a thesis about dependencies. ${ }^{4}$ It limits the factors on which the success of a productive effort may depend to those which make for local differences. No restriction on how those local differences may be

\footnotetext{
${ }^{2}$ Although causing an effect is a sufficient condition for a factor to be responsible for that effect, it is not a necessary condition. Many cases of prevention cannot be thought of as strictly causal, as we are often contemplating the absence of a certain event being responsible for the absence of another. Consider the following case. $T_{1}$ fails to eventuate because life failed to evolve and there are no trees and, thus, no $H_{1}$. Here there is no identifiable causal process involving $H_{1}$ or the production process, but 'because' expresses a relation of responsibility nontheless.

${ }^{3}$ This claim states only a necessary condition for preventing the production of $T_{1}$ from $H_{1}$. No implication of the converse, a sufficient condition for preventing that production, is intended. A productive effort using $H_{1}$ as raw material may result in $T_{1}$ even though there are significant differences from the actual circumstances in the locale of the production. The locality of prevention allows that there is more than one way to produce a table.

${ }^{4}$ Thanks are due to an anonymous referee for bringing the necessity of this clarification to our attention.
} 
made is involved. For instance, it is consistent with the locality of prevention that there be action at a distance, whereby a distant event is directly causally responsible for some effect, e.g., on $H_{1}$, precipitating the failure of $T_{1}$ to emerge. The question of the truth of the locality of prevention is orthogonal to questions about the possibility of action at a distance, and more generally about the nature of causal connections.

The locality of prevention lies at the heart of the present approach. It expresses what looks like a general truth about processes of table-creation: they are essentially local phenomena. The causal-historical path leading to this desk runs through quite specific materials and processes of assembly which are distinct from those leading to your desk, or, indeed, to anything constructed from completely different materials, at other times, or in other places. Because the actual production of this desk from its source hunk is solely a matter of what happens along the causal-historical path, any factor which prevents that production must make a difference along this path. Factors that fail to influence it do not prevent the desk from coming into existence just as it actually does. Running the process which actually leads from $H_{1}$ to $T_{1}$ in the presence of factors which do not locally infringe can still lead to $T_{1}$.

It is important to distinguish two claims at this point. ${ }^{5}$ The claim we defend is that, in the absence of any factor which affects $H_{1}$ or some other element of the production of $T_{1}$, that production may result in the production of $T_{1}$. Nothing prevents $T_{1}$ from being produced, and its emergence is possible. A stronger claim, with which it might easily be confused, is that in the absence of such factors the process must result in the production of $T_{1}$. Nothing prevents $T_{1}$ from being produced, so its emergence is inevitable. The stronger claim, unlike the weaker, articulates a sufficient condition for being $T_{1}$ : any table which is produced from $H_{1}$ under precisely the actual conditions is, as a matter of necessity, $T_{1}$. The present approach eschews such sufficient conditions for being $T_{1}$. All that is promised by the locality of prevention is that $T_{1}$ might still be the product in such a case. But, for all that the principle tells us, it also might not. ${ }^{6}$

The difference between the two claims can be illustrated by appeal to a metaphysical view which accepts the weaker, but denies the stronger. Consider a view according to which there are no non-trivial sufficient conditions for being $T_{1}$. $T_{1}$ 's identity is 'bare' in the sense that it depends on no further facts about either $T_{1}$ or anything else. A proponent of this view holds that $H_{1}$ 's

\footnotetext{
${ }^{5}$ We are again indebted to that same anonymous referee for pointing out the need to highlight this distinction.

'There is a delicate question here about the force of the 'might' in ' $T_{1}$ might still be the product.' Although we find the ordinary English locution expresses our thought tolerably well, those who are overly used to hearing 'could' as 'there exists a possible world in which' may be left puzzled. In the locution of possible worlds, our claim amounts to this: any condition or factor $F$ not affecting the locale of the $H_{1}-T_{1}$ production, but otherwise compossible with it, is such that there is a possible world in which $F$ obtains, and $T_{1}$ is a table produced from $H_{1}$. In that sense the production can still succeed, even though $F$ takes place. Of course this is consistent with there being another possible world in which $F$ obtains and $T_{1}$ is not produced from $H_{1}$.
} 
being in the place that it actually was, being manipulated and shaped as it actually was, with the tools, by the workers, and under the circumstances it actually was, provides no guarantee that $T_{1}$, rather than some other table, is the product. The product of their labors might simply have been a different table. The proponent of bare identities, then, denies the stronger claim that $T_{1}$ must emerge in those circumstances. But she will find nothing to quarrel with in the locality of prevention: it is the inevitability of $T_{1}$ 's emergence she finds repugnant. Its possibility is no problem for her. ${ }^{7}$

This distinction will also help to contrast our claim with another, more familiar thought for which it may be taken. It is sometimes claimed that identity is an 'intrinsic' relation. Expressions of this view vary widely, but the basic thought is that relations of identity hold only in virtue of the (other) intrinsic properties of the relata and cannot be determined by the existence or nonexistence of some other thing or the obtaining of some extrinsic fact. ${ }^{8}$ One version of this thought, the 'only $x$ and $y$ principle,' claims that the identity of a thing with itself over time cannot depend on whether some other individual is or is not present and rules out, for instance, 'best candidate' theories of identity over time. ${ }^{9}$ Although we have formulated our idea in terms of a factor's locality - its making a difference to those objects, events, and agents in the locale of a production process - and not the intrinsic properties of a certain object or region, there are clear affinities between the two ideas. One who accepts the intrinsicness of identity should also accept the locality of prevention, as a world with no local differences in the production process may also be one with no intrinsic differences, and an intrinsic duplicate of that production process will have a product which is intrinsically just like $T_{1}$.

Even so, it should be clear that the claim of intrinsicness goes beyond ours in a number of ways. First, the intrinsicness claim, especially in its 'only $x$

\footnotetext{
${ }^{7}$ We are not endorsing the position according to which there are no non-trivial sufficient conditions for being $T_{1}$. We broach the position only for the purposes of illustration. It does serve to underscore, however, the broad appeal that the locality of prevention has. Some of the literature on the necessity of origin rests the case for origin theses on the rejection of this position, e.g. (Forbes, 1985, ch. 6). Noonan (1983) thinks the case for origin theses rests on the rejection of 'bare' identities (see esp. p. 3), but argues against the ultimate coherence of such a position. Obviously, the route we are sketching here does not rest on its rejection.

${ }^{8}$ We should distinguish three claims that may march under the banner 'the intrinsicness of identity': (1) If $x=y$, then the identity of $x$ and $y$ is an intrinsic feature of $x$ (i.e. $y$ ). (2) Whether $x=y$ is not determined by any merely extrinsic feature of $x$ or $y$. (3) Not only is (2) true, but whether $x=y$ is determined by other intrinsic features of $x$ and $y$. (2) implies (1), for if the identity of $x$ and $y$ were an extrinsic feature of $x$, then an extrinsic feature of $x$, namely its identity with $y$, would determine whether $x$ is identical to $y$. If the existence of a 'best candidate' $z$ for identity with $x$ is held to be an extrinsic feature of $x$ or $y$, then (2) will also yield the 'only $x$ and $y$ principle' mentioned below. A proponent of the 'bare identities' view will find nothing to quarrel with in either claim. Since, according to this view, nothing other than the fact that $x=y$ settles the question of whether $x=y$, its proponent can embrace both (1) and (2). But the third claim is simply inconsistent with the 'bare identities' view. Since proponents of the intrinsicness of identity are so often motivated by a distaste for bare identities, we shall assume throughout that the strongest claim (3) is intended.

${ }^{9}$ For the intrinsicness thesis and the only $x$ and $y$ principle, see, e.g., Wiggins (1980, 94-99) and Noonan (1985b). For the closest continuer theory, see Nozick $(1981,29-70)$ and Noonan (1985a).
} 
and $y$ ' guise, is usually applied to questions of identity over time. We make no claims about what form a correct account of identity over time must take. Our concern is only with what it takes to prevent a given object from coming to be and not with its continued survival after that point. The locality of prevention is compatible with a variety of accounts of identity over time, including 'best candidate' or even a counterpart-theoretic accounts, whether or not such theories are advisable.

This leaves 'cross-world' applications of the intrinsicness claim, which bring us to a second, more important difference. The intrinsicness claim is typically taken to imply a sufficiency principle for cross-world identity: given that $T_{1}$ eventuates from $H_{1}$ in a region $R$ in $w$, any world which is an intrinsic duplicate of $w$ in $R$ (and contains $H_{1}$ in $R$ ) will be a world in which $T_{1}$ eventuates from $H_{1}$ in $R$. (Noonan, 1985b, 82) This is precisely the sort of consequence to which we deny that the locality of prevention need be committed. As should be clear from the example of bare identities, the locality of prevention only requires that $T_{1}$ may eventuate in such a similar situation, not that it must. We emphasize the distinction between 'may' and 'must' here both because weaker premises make stronger arguments and because keeping the distinction firmly in mind will help distinguish the present approach from those based on sufficiency principles when we come to them.

The applicability of the locality of prevention is no less general than its appeal. Nothing about the locality of prevention is really specific to tables. Analogous restrictions on prevention seem to govern the production of most kinds of material objects, many kinds of events, and perhaps even some kinds of nonmaterial objects. ${ }^{10}$ Despite their generality, the restrictions imposed by the locality of prevention are not trivial. There are kinds for which these restrictions do not hold. Call something a prototypical table if it is the first table ever made in the universe. We may prevent the production of a prototypical table from a source hunk simply by constructing another prototypical table at some earlier point in time. Such prevention need not be local. ${ }^{11}$ It is also easy to find examples of kinds for which interference might occur 'after the fact.' Call something a super-prototypical-table if it is the only table ever to exist in the universe. What produces a super-prototypical-table in one circumstance may fail to do so in another. The construction of a second table, even after the first is

\footnotetext{
${ }^{10}$ Although the independence approach to origin theses offered here applies most obviously to material objects, considerations of constitution play no essential role in the reasoning. What matters is rather creatibility and the invulnerability of such creation processes to non-local prevention. Where events and nonmaterial things fit this profile, as, for example, symphonies and species of animal arguably do, similar reasoning will apply. This would appear to vindicate J. L. Mackie's suggestion, (1974, 360), that 'the contrast between the necessity of origin and the contingency of development is not essentially connected with constitution,' while offering a rather different explanation of the contrast.

${ }^{11}$ The interference is not just with the existence of, say, $P T_{1}$, nor with its creation from some hunk or other, but with the production of $P T_{1}$ from, say, $H_{1}$. We are thinking of productions as individuated in part by the kind of thing the product is. So, supposing $P T_{1}$ to be a prototypical table constructed from $H_{1}$, the production of that very table from $H_{1}$ may fail in some other world, even though the right table is produced from $H_{1}$ in that world. The prototypical table production fails because the product is not a prototypical table.
} 
completed, spoils it. Productions of prototypical- and super-prototypical-tables are vulnerable to forms of competitive interference. What prevents their production is our running the very same sort of process on another hunk somewhere else.

\section{Independence Principles}

The contrast with ordinary tables is instructive, for ordinary tables do not seem vulnerable to the same sort of non-local, competitive interference. As far as making $T_{1}$ from $H_{1}$ goes, it just doesn't matter what you do with some other hunk of wood somewhere else in the universe. Make it into a table or don't. As long as the second process doesn't infringe on the process which actually makes $H_{1}$ into $T_{1}$, this process may well run as it actually did, resulting in $T_{1}$. The reasoning is symmetric. Suppose we do make that second hunk into a table. Whether we make $H_{1}$ into $T_{1}$ or not is irrelevant to the success of our new endeavor, unless the two processes locally interfere with one another.

The locality of prevention has the following consequence: if one table production need not have effects in the locale of another and vice versa, then it is possible for both productions to succeed. Table productions can be isolated from the effects of other table productions in most cases. The upshot is that processes that turn hunks into tables seem to enjoy a form of independence from one another. A process that turns one hunk into a table need not interfere with any other, though there are cases in which they do, in fact, interfere. Suppose we burn one of the hunks in order to power the machine which makes the other hunk into a table. In this situation, we can no longer make any of the tables we might otherwise have made from the burned hunk. But this connection between the processes is contingent. Had we found another source of power, the second hunk would have remained available for table-manufacture. The relevant form of independence is one which rules out only necessary interference between table-making processes.

If this reasoning is correct, we seem to have the following situation. Given any two distinct hunks, a table constructed from the first hunk can, in principle, also be constructed in the presence of the production of any of the tables which can be constructed from the second hunk. This is what we call an independence principle. $^{12}$ It expresses the compossibility of table-productions from distinct hunks. Independence is the ineluctable result of the locality of prevention. Because making a table is just a matter of what happens locally along the casual-historical path, the paths are compossible when nothing requires one to affect the other as a matter of necessity. Whenever processes of production

\footnotetext{
${ }^{12}$ Independence principles, and the availability of valid arguments from them to origin theses, appear to have been first noticed by Kit Fine and Robert Stalnaker. In footnote 11 of (Salmon, 1979), Salmon attributes a similar idea to Fine and Stalnaker, but does not there recognize that it represents an approach to origin theses fully distinct from his own. Later writers appear either to have overlooked the suggestion or to have accepted Salmon's assimilation of the suggestion to the sufficiency approach. If the interpretive suggestion of note 1 is correct, the idea is originally Kripke's.
} 
are invulnerable to non-local prevention, there will be an analogous principle of independence. So, independence principles seem to hold no less generally than does the locality of prevention. ${ }^{13}$

In fact, there is reason to think that independence principles can hold even in the presence of certain failures of the locality of prevention. The only failures of locality which undermine independence principles are those in which non-local competitive prevention is possible, as in the case of the prototypicaltables. While we believe that the case for independence is clearest for the broad array of processes which are plausibly thought immune to all forms of non-local prevention, it is logically possible for a pair of production processes to be independent in the required sense even though each is vulnerable to some forms of non-local prevention. This means that the independence approach to origin theses may remain available in some difficult cases, though at the cost of additional philosophical work. Although it is our purpose here to illustrate the core of the approach, a brief discussion of an example may indicate the direction one might take here.

Our example is inspired by the literature on personal identity. Our focus, however, will be on the production of human beings rather than persons. Suppose a human being, $H_{1}$, actually develops from gametes $G$. Suppose also that it is possible for the process which actually led to $H_{1}$ to have led to a human being $H_{2}$, who later 'split,' resulting in identical twins. Consider a view which maintains, "It is essential to $H_{1}$ that she not split. Because of the split, $H_{2}$ and $H_{1}$ are distinct; in general it is impossible for a human being to split like this if she does not actually do so. In effect, $H_{2}$ 's split prevents the productive process involving $G$ from yielding $H_{1}$." If we accept this view, we seem to have a case of non-local prevention par excellence. An event in the subsequent history of the product $H_{2}$ prevents the production from yielding $H_{1}$, but not by any effect on $G$ or other elements of the process. ${ }^{14}$

Even granting this view, it remains clear that the production processes display the relevant form of independence from production processes involving other gametes. The splitting of an individual coming from a set of gametes is not required by the coming of an individual from distinct origins. $\mathrm{H}_{2}$ 's production from $G$ is as compossible with the production of individuals from other origins as $H_{1}$ 's is. ${ }^{15}$

Independence principles imply that, even if one thinks that a given origin

\footnotetext{
${ }^{13}$ The 'seem to' should be taken seriously. We will meet examples where independence principles fail even though locality holds when we discuss productions whose source-hunks overlap below. Such cases are very much the exception rather than the norm.

${ }^{14}$ This view is broached for purposes of illustration only. We do not endorse the view, and will make no attempt to motivate or defend it. The philosophical literature on personal identity is replete with views regarding the metaphysics of the fission of persons. Perhaps a good place to start is (Parfit, 1986).

${ }^{15}$ We bypass for now issues involving the entanglement of productive processes that lurk in the wings here. Consider, for instance, cases of productions from overlapping source-hunks, a possibility for gametes. It is doubtful that the production of $H_{1}$ from $G$ is compossible with the production of another human being from overlapping origins. These issues do not affect the paradigm cases for origin theses, where there is no such entanglement, and will be discussed in detail later.
} 
could have given rise to a variety of different individuals — distinct solo alternatives, twins, or what have you - all the processes starting with that material origin are independent of those starting with distinct material origins; any of the individuals obtainable from one are compossible with any of the individuals obtainable from another. Perhaps $H_{1}$ can only appear when there is no splitting, but it may appear alongside any individual from another origin. Independence principles govern relations between productions from distinct source hunks. Since the splitting cases do not involve other source hunks, they do not touch independence principles, even if they threaten the locality of prevention. ${ }^{16}$

\section{The Argument from Independence}

We are now in a position to understand origin theses, not as bottom level metaphysical principles or mere intuitions, but instead as byproducts of independence principles. Let us start with an explicit characterization of independence for tables. Here and throughout the paper, we use 'made from' as short for the relation made entirely and exclusively from, i.e. that relation which holds between tables and hunks of material that contain all and only the material from which the table is made. ${ }^{17}$

(T-IND) Necessarily, given a table, $T_{1}$, made from a hunk, $H_{1}$, for any table, $T_{2}$ which might be made from a hunk, $H_{2}$, distinct from $H_{1}$, it is also possible that both $T_{1}$ is a table made from $H_{1}$ and $T_{2}$ is a table made from $\mathrm{H}_{2}$.

In line with our reasoning, this expresses a compossibility claim about processes of table production. As promised, it is no logical truth, for the inference

\footnotetext{
${ }^{16}$ Another issue on which independence does not pronounce is whether the twins resulting from the split of $\mathrm{H}_{2}$ are separately producible. Independence only maintains that individuals having distinct material origins are compossible, but the twins share a material origin. Furthermore, independence principles only license inferences from claims about what is separately producible to what is jointly producible, never the reverse.

${ }^{17} \mathrm{It}$ is natural in English to speak of tables being made from hunks containing only a portion of their original material, as in 'this table was originally made from that leg.' If we allowed such a colloquial understanding of the expression 'made from', then the resulting origin theses would have actual counter-examples. For instance, this table was not only "made from" that leg, but also from other, distinct legs. It is not only possible for this table to have been "made from" a hunk distinct from this leg, it actually was "made from" a distinct hunk, e.g. that other leg. We adopt the stipulation of the main text as a way of avoiding such uninteresting counter-examples. One could, alternatively, avoid the irrelevancies by taking the relation indicated colloquially by "made from" as one's starting point in formulating our principles. One could then specify the more artificial relation we intend as one that holds between a table and a hunk iff all and only parts of the hunk are things the table is "made from", in the colloquial sense. (Really, an even more complicated specification is required, given that it is also natural in English to speak of a table's being made from a hunk which is only partially used up in the manufacture, as in 'this table was originally made from a certain block of wood, half of which was left over'.) Because substituting this specification for 'made from' would overcomplicate the formulation of our principles, we reserve the expression 'made from' for the relation made entirely and exclusively from, as we explain in the main text.
} 
from 'Actually $\mathrm{P}$ ' and 'Possibly Q' to 'Possibly $\mathrm{P}$ and Q' is invalid. Furthermore, we believe that (T-IND), as it stands, is susceptible to counter-example. Because our purpose here is to demonstrate a valid argument schema from independence-style principles to origin theses and not a full investigation of the truth of such principles, it is simplest to consider an unrestricted version of independence. Later, we will suggest how the principle might be restricted in order to restore its plausibility.

The argument requires two other premises. The first is a familiar logical principle, the (necessary) necessity of distinctness.

(ND) Necessarily, if $x \neq y$, then necessarily $x \neq y$.

The second is another metaphysical principle. Call it origin uniqueness.

(OU) Necessarily, if $T_{1}$ is a table made from $H_{1}$ and $T_{2}$ is a table made from $H_{2}$ and $H_{1} \neq H_{2}$, then $T_{1} \neq T_{2}$.

Keeping in mind our stipulative use of 'made from,' this principle says that a single table cannot entirely and exclusively originate from each of two distinct hunks within a single possible world. Suppose that there is a world in which $T_{1}$ is made from both $H_{1}$ and $H_{2}$. Our stipulation regarding the use of 'made from' requires that $H_{1}$ and $H_{2}$ contain exactly the same material. Barring the possibility of distinct but exactly coincident hunks, $H_{1}$ and $H_{2}$ are identical, as (OU) says. The principle is no logical truth, since the impossibility of distinct but exactly coincident hunks is no logical truth. Nevertheless, (OU) is still a trivial truth about material objects like tables: tables with distinct origins (in a world) are also distinct. The conclusion is a necessity of origin thesis for tables.

(T-NO) Necessarily, given a table, $T_{1}$, made from a hunk, $H_{1}$, any table, $T_{2}$, which might be made from a hunk, $H_{2}$, distinct from $H_{1}$, is distinct from $T_{1}$.

Now the argument. Start with a table, $T_{1}$, made from a hunk, $H_{1}$. Let $T_{2}$ be some arbitrary table it is possible to make from a hunk, $H_{2}$, distinct from $H_{1}$. Since $T_{1}$ actually comes from $H_{1}$ and it is possible that $T_{2}$ come from $H_{2}$, the independence principle says that both are jointly possible in some world $w$. Since $H_{1}$ and $H_{2}$ are distinct, they are distinct in $w$ as well by the necessity of distinctness. By origin uniqueness, the distinctness of the hunks in $w$ shows that $T_{1}$ is distinct from $T_{2}$ in $w$. An application of the necessity of identity shows that $T_{1}$ and $T_{2}$ are actually distinct as well. Since the choice of $H_{2}$ and $T_{2}$ was arbitrary, we conclude that any table it is possible to make from such an $H_{2}$ is distinct from $T_{1}$. Since the choice of starting world was arbitrary, the conclusion holds for all possible worlds. Q.E.D. ${ }^{18}$

\footnotetext{
${ }^{18}$ The proof is straightforward and requires only the $\mathrm{K}$ and $\mathrm{T}$ axioms along with the rule of necessitation. One could, for elegance, use B and the necessity of identity in lieu of (ND). The relevant QML symbolizations are:$$
\text { T-IND } \square \forall t_{1} \forall h_{1}\left(O\left(h_{1}, t_{1}\right) \Rightarrow \square \forall t_{2} \forall h_{2}\left(h_{1} \neq h_{2} \wedge O\left(h_{2}, t_{2}\right) \Rightarrow \diamond\left(O\left(h_{2}, t_{1}\right) \wedge O\left(h_{1}, t_{1}\right)\right)\right)\right)
$$$$
\text { ND } \square \forall x \square \forall y \square(x \neq y \Rightarrow \square(x \neq y))
$$ 


\section{Circularity Worries}

One natural reaction to the argument is to think that, while valid, it simply begs the question because the independence principle just is the necessity of origin in disguise. What difference is there, one might ask, between being told that a table could have come from no other hunk and being told that the production of any table from another hunk is compossible with the first table? Strictly speaking, the charge is false. Independence and the necessity of origin do not imply one another. First, independence, by itself, does not imply any origin thesis in the absence of the necessity of distinctness and origin uniqueness. For instance, if distinctness were contingent, then $T_{1}$ could be made from $\mathrm{H}_{2}$ in a world in which that hunk is identical to $H_{1}$. While neither the necessity of distinctness nor origin uniqueness is open to much doubt, our reasons for accepting them come from quarters far removed from those which support the origin thesis. Second, the necessity of origin does not imply independence. Even if no table could ever come from other matter, it might still be the case that independence fails because some distinct table-productions are not compossible. The necessity of origin only denies the existence of certain possibilities, while independence principles make a positive claim that a certain situation, that containing both table-productions, is possible.

Such logical niceties aside, one might still think there is something to the spirit of the charge. After all, if independence is true, it rules out any form of necessary interference between the making of $T_{1}$ from $H_{1}$ and the making of any table at all from $H_{2}$. Haven't we just stipulated away the apparent counter-example to the origin thesis, that in which the interference comes from our making $H_{2}$ into $T_{1}$ ? To see why the answer is 'no,' we need to go back to the justification of independence. Where $H_{1}$ and $H_{2}$ are distinct hunks and we have a way making $H_{1}$ into a particular table, if we also have a way of making $H_{2}$ into a particular table, then it seems that we could, in principle, run both of these processes together. The distinctness of the hunks seems to guarantee that there is no necessary interference between the processes; in at least one world, we can run them both and get the very tables we produced separately. Someone who wants to claim that we can make $H_{2}$ into $T_{1}$ needs to explain either why we could not also run the process which in fact turned $H_{1}$ into $T_{1}$ or why that process could not result in $T_{1}$. Either sort of explanation would appear to violate the locality of prevention. It won't do simply to say, 'We've already made $T_{1}$, so $T_{1}$ can no longer be made,' without also telling us what factor necessarily affects $H_{1}$ or some other element needed to produce $T_{1}$ from it. Without some explanation of why the two processes must interfere with each other, the objector is left baldly claiming some unspecified form of interference. One may object to independence, but the assertion of independence is not just the bald assertion of the origin thesis. Independence has its own grounds of

OU $\square \forall t_{1} \forall h_{1} \square \forall t_{2} \forall h_{2} \square\left[\left(h_{1} \neq h_{2} \wedge O\left(h_{1}, t_{1}\right) \wedge O\left(h_{2}, t_{2}\right)\right) \Rightarrow t_{2} \neq t_{1}\right]$

T-NO $\square \forall t_{1} \forall h_{1}\left(O\left(h_{1}, t_{1}\right) \Rightarrow \square \forall t_{2} \forall h_{2}\left(h_{1} \neq h_{2} \wedge O\left(h_{2}, t_{2}\right) \Rightarrow t_{2} \neq t_{1}\right)\right)$ 
support, and one who objects to the origin thesis must find some flaw in these independent grounds.

\section{Independence and Branching Times}

It will be worthwhile at this point to distinguish the independence approach to the origin theses from the two other dominant approaches, the branching account and the sufficiency account. The former grounds origin theses in the so-called branching times model of necessity. Questions about possibility are settled according to this model by asking whether the history of the universe could have gone as it actually has, up to a point, and then diverged in a way that brought about the possibility in question. On one simple version of this framework, the de re possibilities for $T_{1}$ are given in all the ways the universe could have gone on after the advent of $T_{1}$. None of these histories, however, include $T_{1}$ 's coming from another hunk. By the time of the divergence, it is too late for $T_{1}$ to have had a different origin. There are possibilities for tables included in earlier divergences, but none of these, according to the framework, are possibilities for $T_{1}$; these divergences are too early to represent possibilities for it.

The branching times and independence approaches differ, most obviously, in the scope of the claim in which they ground their arguments. While the independence approach relies on a specific insight about what it takes to prevent the production of a material object, the branching times approach relies on an overarching claim about what, in general, determines the possibilities for things, in short, a full-fledged interpretation of necessity. The independence approach, in contrast, requires no particular interpretation. The two approaches may also conflict directly, for it is not obvious that independence principles will come out true under a branching times interpretation of necessity. Consider, for instance, the simple version of the branching times interpretation broached in the last paragraph. Let $T_{2}$ be a merely possible table which might have been created from $H_{2}$ long before $T_{1}$ was actually created from $H_{1}$ and thus resides on a branch which diverges from actuality prior to the advent of $T_{1}$. Compresence with the production of $T_{2}$ from $H_{2}$ is not a possibility for $T_{1}$ as independence claims, since only branches which diverge from actuality after $T_{1}$ 's advent represent possibilities for it. Perhaps some subtler, more complicated version of the branching times model is consistent with independence principles. Even so, the subtlety and complexity required underscores the differences between the two approaches.

\section{$7 \quad$ Independence and the Sufficiency of Origin}

The difference between the independence approach and the sufficiency approach is more subtle because the forms of argument are superficially similar. Both use the necessity of distinctness, origin uniqueness, and some compossibility claim 
about table productions to reach the origin thesis. The sufficiency account starts with an independence-like principle, though one that is weaker and more obviously true. Call it weak-independence for tables.

(T-WIN) For any two distinct hunks of matter, $H_{1}$ and $H_{2}$, and any table, $T_{1}$, made from $H_{1}$, it is possible both that $T_{1}$ is a table made from $H_{1}$ and that $T_{2}$ is a table made from $\mathrm{H}_{2}$.

Of our usual $H_{1}-H_{2}-T_{1}$ situation, this says that it is possible to construct some table or other from $H_{2}$ alongside $T_{1}$; call it $T_{2} . T_{2}$ is, by the necessary distinctness of the hunks and origin uniqueness, distinct from $T_{1}$ and necessarily so. But this leaves open the possibility of another, special table, $T_{3}$, which is constructible from $H_{2}$ but is not compossible with $T_{1}$ 's production from $H_{1}$. Thus, the logic of the situation so far allows that $T_{3}$ might be $T_{1}$, and thus a counter-example to the origin thesis. This gap is bridged by appealing to another principle, the sufficiency of origin for tables.

(T-SO) If it is possible for a table, $T$, to originate from a hunk, $H$, then necessarily any table originating from $H$ is $T$.

This claims that each hunk has lurking in it at most one possible table, or, more generally, that having a particular origin is a sufficient condition for being a particular table. With the sufficiency principle in hand, one may infer that $T_{3}$ is identical to $T_{2}$ because both share the very same origin, viz., $H_{2}$. Since it was shown that $T_{2}$ is necessarily distinct from $T_{1}$, we can conclude the same for $T_{3}$ and for any table coming from a distinct hunk.

We, along with many others, are skeptical about the truth of such sufficiency principles, ${ }^{19}$ but what is relevant here is that the argument from independence does not presuppose, and is compatible with the falsity of, the sufficiency of origin. The independence principle says that the making of $T_{1}$ from $H_{1}$ does not foreclose the possibility of making any table that might otherwise be made from $\mathrm{H}_{2}$, for both may be constructed in the same world. The truth of this claim is entirely compatible with our being able to make a number of alternative tables from a single hunk of matter, and this is the denial of the sufficiency principle. Essentially, the sufficiency principle functions in the argument by ensuring an unusually strong form of independence between table-makings: no table from $H_{2}$ need interfere with $T_{1}$ 's coming from $H_{1}$ because any table from $H_{2}$ is $T_{2}$, which we already know from (T-WIN) does not interfere. But once one sees how the argument from independence proceeds, it becomes clear that sufficiency principles are an unnecessarily strong way of guaranteeing the independence required to derive the origin thesis.

While it should be clear that the argument from independence does not rely on any sufficiency of origin principle, one might think that sufficiency reasoning is still at work in the justification of independence itself. ${ }^{20}$ This impression may

\footnotetext{
${ }^{19}$ For some doubts, see, e.g., Salmon (1979); Robertson (1998); Hawthorne and Gendler (2000); MacKay (1986); Chihara (1998); Della Rocca (1996); Sarkar (1982); Noonan (1983); and Kripke (1980, 43, 46).

${ }^{20}$ Teresa Roberston emphasized the need for this point.
} 
be dispelled. We suggested that independence principles have their source in the principle of locality of prevention: a production process can be prevented only by factors which affect the raw materials, workers, tools, and facilities involved. Where there are two such processes which need not affect one another, they are compossible because nothing prevents both from occurring together in some world. One way of understanding this reasoning would invoke a sufficiency principle for processes. If one thought that the process which actually leads from $H_{1}$ to $T_{1}$ would inevitably lead to $T_{1}$ 's emergence, then any world containing this unaltered process alongside some other such process is a world containing $T_{1}$ and some other table.

We have emphasized that, while one may reason in this fashion, one need not. Securing the truth of independence for a pair of non-interfering processes, say those which take $H_{1}$ into $T_{1}$ and $H_{2}$ into $T_{2}$, requires only that there be some world in which we can run them both with these results. It is a matter of indifference whether there are some other worlds in which running these processes leads to tables other than $T_{1}$ and $T_{2}$. It is for this reason that we say that running the process which actually leads from $H_{1}$ to $T_{1}$ in the presence of another process which doesn't locally infringe can lead to $T_{1}$, not that it must. Once again, sufficiency reasoning turns out to be stronger than is required for the argument. We might also note that questions about the necessary features of processes are bypassed on our approach, for it is again a matter of indifference whether, say, the process which actually led from $H_{1}$ to $T_{1}$ could have run differently with the same result or, if so, how differently it could have run. Given a pair of hunk-to-table processes, all that matters is the possibility of running them unaltered and getting those same tables in at least one world.

\section{Overlapping Origins}

(T-IND), as stated, is not problem-free. It turns out to be counter-intuitive when $H_{1}$ and $H_{2}$ have much of their material in common. If $H_{1}$ is made into a table in such a case, then there may not be enough of $H_{2}$ left to craft some table we might have otherwise obtained; the two tables compete for raw materials. Such cases are also ones in which a necessity of origin thesis is implausible. Couldn't $T_{1}$ have been made from slightly different matter? Couldn't it therefore have been made from a hunk distinct from $H_{1}$, but sharing much material in common? If our claim that independence principles ground origin theses is correct, then it is no coincidence that origin theses seem implausible in cases where independence fails. Such cases also suggest that we may restore (T-IND)'s plausibility by restricting it, from distinct hunks, to non-overlapping hunks.

RT-IND Necessarily, given any two non-overlapping hunks, $H_{1}$ and $H_{2}$, and a table, $T_{1}$, made from $H_{1}$, for any table, $T_{2}$, that might be made from $H_{2}$, it is also possible that both $T_{1}$ is a table made from $H_{1}$ and $T_{2}$ is a table made from $\mathrm{H}_{2}$. 
Such a restricted (T-IND) would support a similarly restricted and more plausible origin thesis, like that defended by Kripke, Salmon, and others, that no table could have been constructed from a hunk entirely disjoint from that table's actual originating hunk.

A second sort of case appears to be a counter-example to even this restricted form of independence and complicates our defense of origin theses. Suppose that $H_{1}$ is a block of petrified wood which comes from $H_{2}$ by petrification, a process in which all the organic matter of $\mathrm{H}_{2}$ is replaced by minerals. ${ }^{21}$ Once again, making a table from $H_{2}$ will preclude our making any tables from $H_{1}$, despite the fact that the two hunks share no matter. Making some $T_{2}$ from $\mathrm{H}_{2}$ and allowing $H_{2}$ to petrify into $H_{1}$ compete for raw materials. $H_{2}$ cannot both be made into the table $T_{2}$ and remain available for petrification into the hunk $H_{1}$. So (RT-IND) is evidently false in this situation.

There are two issues here. The first is the general truth of independence-style principles. Even though $H_{1}$ and $H_{2}$ do not overlap in the sense of sharing matter, it is clear from the perspective of the locality of prevention principle what is going on. The processes which lead from $H_{2}$ to $T_{2}$ and from $H_{2}$ through $H_{1}$ to $T_{1}$ overlap in their causal histories and necessarily interfere with one another. This suggests that (RT-IND) does not exhaust the content of the locality of prevention principle and that one could develop a notion of 'non-overlap' other than 'disjointness of matter' which would validate independence principles. Since it is not our primary aim to arrive at the truth about independence principles, we will not pursue this suggestion here. The second issue, more important in this context, is the impact of such examples on the plausibility of origin theses. Even if there is such a sense of 'non-overlap' which validates independence, it looks as if there is no origin result in the offing with respect to some items in a object's causal past, those whose role in the causal chain wholly consumes them as a matter of necessity (so not, for instance, one's grandmother). Even if such a version of independence remains true, it will support only a weaker origin thesis, one which does not foreclose the possibility that $T_{1}$ originate from a hunk whose causal history is suitably entangled with $H_{1}$ 's. In these cases, a skeptic might claim that the independence approach must allow that $T_{1}$ could have come from $H_{2}$ instead of $H_{1}$.

It is important to be clear on what sort of skepticism these cases might be thought to encourage. Such cases cannot support a broad skepticism about origin theses for two reasons. First, there remain many source-product pairs for which instances of the independence principle are obviously true, and so corresponding instances of origin theses will be true of them. This delivers the paradigmatic cases of origin theses, where there is no entanglement between productive efforts: this table could not have been made of some other hunk of wood grown in Australia, no matter how similar; nor could Elizabeth Windsor have come out of Harry and Bess Truman's gametes. Second, the coherence of examples like the petrified wood case turns out to presuppose the acceptance of some origin theses. Consider how it is plausible to claim that the petrification

\footnotetext{
${ }^{21}$ This case is due to James Forrester.
} 
of $H_{2}$ into $H_{1}$ necessarily competes for raw materials with the production of $T_{2}$ from $H_{2}$. There will be necessary competition only if $H_{1}$ could not have come from any hunk other than $\mathrm{H}_{2}$. That is, the skeptic uses an origin thesis about hunk-productions to arrive at a counter-example to independence principles for table-productions. If our skeptic's aim is to undermine the plausibility of origin theses in general, then her position is not coherent.

There remains a rather different sort of 'skeptic,' one who endorses the origin thesis in a wide range of cases, including, here, the impossibility of $T_{1}$ 's coming from $\mathrm{H}_{2}$. Such a skeptic does not deny that independence arguments give rise to origin theses, but challenges our claim that independence is the full story of the conceptual grounds of origin theses. It is, however, unclear to us that this is genuinely impossible or that the independence approach gives the wrong answer in this case. Suppose, for instance, that the craftsmen who actually made $T_{2}$ from $\mathrm{H}_{2}$ were rather more indolent and allowed the wood from $\mathrm{H}_{2}$ to petrify in the course of their activity. Might not the product of their admittedly languid efforts have been $T_{1}$ ? This would seem to be a case in which the stone table $T_{1}$ might be produced from the wooden hunk $H_{2}$. There is thus some temptation to treat this case as analogous to the earlier case of overlap: because the origins are entangled, independence fails and so does the necessity of origin. It is true that the 'overlap' is more generally spatio-temporal-causal in nature than the mere sharing of matter, but there was already need for this generalization.

We believe that it is difficult to pronounce on purported cases of the origin thesis in a vacuum. Indeed, the point of any of the approaches to origin theses is to ground them in more than fleeting intuitions. Our imagined opponent thinks $T_{1}$ could not have originated from $H_{2}$. Could she appeal to either of the other two approaches to support her case? The sufficiency approach will not help. The petrification case is one in which even weak-independence fails. Not even one table can be made from $H_{1}$ in the presence of a $T_{2}$ made from $H_{2}$, contra (T-WIN). So the sufficiency approach does not show that $T_{1}$ could not have been made from $\mathrm{H}_{2}$. The simple branching account appears to support the case, as $T_{1}$ and $T_{2}$ lie, as it were, on different temporal branches. The simple version, however, has problems precisely with avoiding making the time and circumstances of creation a necessary feature of objects. ${ }^{22}$ Presumably one might avoid these difficulties by providing a more sophisticated account which achieves plausibility by allowing branches before the time of creation. Once we are permitted to take liberties with the circumstances that predate $T_{1}$ 's advent, however, it is more difficult to resist our little story about the indolent craftsmen. It would seem at first blush that a more liberal version of the branching times approach faces the same difficulties in handling the case of petrification as do the independence and sufficiency approaches. This is only a first impression. It is difficult to know whether or how a sophisticated branching times approach might support an origin result in the petrification case without being in possession of the details. But there is, as yet, no compelling reason to abandon the claim that independence results alone ground origin theses.

${ }^{22}$ See Penelope Mackie (1998) for a clear and sustained argument for this point. 


\section{Conclusion}

One measure of the strength of a philosophical approach is the questions it allows one to frame and the resources it provides for answering them. The independence approach shows such strength as one moves beyond the model case of tables. The plausibility of origin theses turns on a host of further questions about the truth and scope of independence principles: Are there ways, other than competition for raw materials, that creation processes can compete, giving rise to necessary interference? What about competition for time, place, or agent of creation? For what kinds of objects do we get independence principles? Are they all material? Do they all involve initial constitution? Not every kind will support such a principle, as the prototypical-table example shows, but what is the widest class? Is it philosophically interesting? The independence approach, in turn, suggests that we may answer such questions by considering the source of independence principles, the invulnerability of creation processes to non-local prevention.

Despite such questions, we draw two firm conclusions. First, there is a valid, non-circular argument from independence principles to origin theses. Second, the independence approach is distinct from both branching and sufficiency approaches and does not rely on their primary assumptions. What is more interesting, and more tentative, is the idea that independence principles derive from a more basic and general truth about processes of creation, that they are essentially local phenomena. From this perspective, origin theses are mere consequences of what has the look of a genuinely basic metaphysical truth. We believe that the independence approach offers a novel and insightful framework for thinking about origin theses and, ultimately, for assessing their truth. ${ }^{23}$

\section{References}

Charles Chihara. The Worlds of Possibility. Clarendon Press, Oxford, 1998.

Michael Della Rocca. Essentialism: Part 2. Philosophical Books, 37:81-9, 1996.

Graeme Forbes. On the philosophical basis of essentialist theories. Journal of Philosophical Logic, 10:73-99, 1981.

Graeme Forbes. The Metaphysics of Modality. Oxford University Press, Oxford, 1985.

John Hawthorne and Tamar Szabó Gendler. Origin essentialism: The arguments reconsidered. Mind, 109:285-98, April 2000.

\footnotetext{
${ }^{23}$ The ideas for this paper first emerged during a seminar at UCLA on Lecture 3 of Naming and Necessity in the Fall of 1999. We thank all of the participants in this seminar for their help. Special thanks are due to Joseph Almog, David Kaplan, Collin O'Neil, and Dominik Sklenar, for helping to ease this paper's birth-pangs. We also would like to thank Kit Fine, James Forrester, Paul Hovda, D.A. Martin, Nathan Salmon, Teresa Robertson, Takashi Yagisawa, Byeong-Uk Yi, and two anonymous referees for their helpful comments on various drafts and presentations.
} 
Patricia Johnston. Origin and necessity. Philosophical Studies, 32:412-18, 1977.

Saul Kripke. Naming and Necessity. Harvard University Press, Cambridge, 1980.

Thomas MacKay. Against constitutional sufficiency principles. In Peter French, Theodore Uehling, Jr., and Howard Wettstein, editors, Midwest Studies in Philosophy, Vol. XI: Studies in Essentialism, pages 295-304. University of Minnesota Press, 1986.

J. L. Mackie. De what re is de re modality? Journal of Philosophy, LXXX: 551-61, 1974.

Penelope Mackie. Identity, time, and necessity. Proceedings of the Aristotelian Society, 98:59-78, 1998.

Colin McGinn. On the necessity of origin. Journal of Philosophy, LXXI:127-35, 1976.

Harold Noonan. The necessity of origin. Mind, 92:1-20, 1983.

Harold W. Noonan. The closet continuer theory of identity. Inquiry, 28:195-229, 1985a.

Harold W. Noonan. The only x and y principle. Analysis, 45:79-83, 1985b.

Robert Nozick. Philosophical Explanations. Belknap Press, 1981.

Derek Parfit. Reasons and Persons. Oxford University Press, 1986.

Teresa Robertson. Possibilities and the arguments for origin essentialism. Mind, 107:716-25, October 1998.

Nathan Salmon. How not to derive essentialism from the theory of reference. Journal of Philosophy, LXXVI:703-25, 1979.

Husain Sarkar. Origins and identities. Australasian Journal of Philosophy, 60: 140-51, 1982.

David Wiggins. Sameness and Substance. Harvard University Press, 1980. 